American Journal of Pharmacology and Toxicology 6 (4): 119-123, 2011

ISSN 1557-4962

(C) 2011 Science Publications

\title{
Antimicrobial and Wound Healing Potentials of Vitis Vitigenia Leaves
}

\author{
${ }^{1}$ Krishna Murti, ${ }^{1}$ Manish Kaushik, \\ ${ }^{2}$ Aditi Kaushik and ${ }^{1}$ Deepika Paliwal \\ ${ }^{1}$ Department of Pharmacology, \\ PDM School of Pharmacy, Safidon (Haryana), India \\ ${ }^{2}$ Department of Pharmacology and Chemistry, \\ PDM College of Pharmacy, Bahadurgarh, (Haryana), India
}

\begin{abstract}
Problem statement: The investigation for possible antimicrobial and wound healing activity of ethanolic and aqueous extracts of $V$. Vitigenia L. leaves were performed. The antimicrobial activity of extract was studied against the 8 bacterial and 2 fungal strains using agar cup plate method. Approach: The wound healing effect of both the extract of $V$. Vitigenia L. leaves were evaluated by incision and excision model in rats. The incision model was assessed by the breaking strength and excision model by \% wound contraction and period of Epithelialization. Results: The results showed that both the extracts exhibited significant antimicrobial activity against all the tested microorganisms and have significant wound healing activity as evident from the breaking strength; \% wound contraction and period of epithelialization. Conclusion: Therefore, the present study provides a scientific rationale for the traditional use of $V$. Vitigenia $L$. in the management of wounds.
\end{abstract}

Key words: Various disorders, pharmacological investigations, specimen receipt, healing property, drug development, antimicrobial activity, enough scientific data

\section{INTRODUCTION}

Herbal drugs play a role in the management of various disorders; most of them speed up the natural healing process of humans. Numerous medicinal plants and their formulations are used for various disorders in ethno medical practices as well as traditional system of medicine in India. V. Vitigenia L. is large woody deciduous climber and is frequent in the deciduous forests in India (Basu and Kirtikar, 1999). V. Vitigenia L. is included in the group of fruit drugs (phalavarga) by Charaka. The fruit is antiscorbutic, astringent, carminative, cardio tonic, cooling, emollient and used in anorexia, colic dyspepsia, heart diseases, piles, difficult micturation, skin diseases, thirst and ulcer. A wound may be defined by (Cotran et al., 1999) as a break in the epithelial integrity of the skin or may also be defined as a loss or breaking of cellular and anatomic or functional continuity of living tissue. Wound healing studies are mainly aim to detect various means and factor influencing healing process, so they could be either used or avoid in clinical practice to favorably alter the healing process as stated. Review of literature revealed that the wound healing property of this plant has not been subjected to scientific evaluation and there is not enough scientific data to support the claims made in the ancient literature. The need for safer and effective wound healing drug and the lack of enough scientific data to support the claims made in ancient literature prompted the present study. Since the leaves are one of the important parts of the plant $V$. Vitigenia L., it was used for the pharmacological investigations.

\section{MATERIALS AND METHODS}

Collection and authentication of plant material: Plant of $V$. Vitigenia collected from road side between Shimla and Solan (Himachal Pradesh), India. The collection was done in the month of August. It has been authenticated by Prakash Singh, Reader, Dept. of Botany, A.N, College, Patna, Bihar, India. The specimen receipt was submitted in the department for future reference.

\section{Preparation of the leaves extract:}

Ethanolic extract of $\boldsymbol{V}$. Vitigenia leaves: The leaves were removed from plant and dried in shade then were powdered mechanically to get the coarse powder. 
Weighed quantity of coarse powder (1 kg) was extracted with ethanol at $60-70^{\circ} \mathrm{C}$ up to $72 \mathrm{~h}$. in soxhlet apparatus. A brown semi-solid extract was obtained after concentrating ethanolic extract.

Aqueous extract of $\boldsymbol{V}$. Vitigenia roots: The fresh leaves were collected, powdered and dried under the shade. The aqueous extract was prepared by using maceration technique of extraction. Filter the filtrate. The filtrate was concentrated on water bath using petridish. The temperature was maintained at $50^{\circ} \mathrm{C}$. The semisolid extract was dried and weighed. The semisolid mass (brown colour) was obtained.

Phytochemical screening: Preliminary phytochemical screening of the ethanolic and aqueous extracts of $V$. Vitigenia L. leaves was performed to test the presence of the active chemical constituents. (Anonymous, 1994; Khandelwal, 2008).

Antimicrobial activity: For antimicrobial activity 4 $\mathrm{Gm}+\mathrm{ve}, 4 \mathrm{Gm}$-ve and 2 fungal strains are used.

Gram-positive bacteria: Bacillus subtillis, Staphylococcus aureus, Bacillus Cereus, Micrococcus luteus.

Gram-negative bacteria: Escherichia coli, Proteus vulgaris, Pseudomonas aeruginosa,Salmonella typhi

\section{Fungi: Aspergillus niger, Candida albicans Minimum Inhibitory Concentration (Zone of Inhibition):}

- Agar cup plate diffusion method

- For Gm+ve and Gm-ve Bacteria: Agar Media is used

- For Fungal Strain: Saboraud dextrose agar media is used

\section{Preparation of the tested organisms}

Preparation of standard bacterial suspensions: The average number of viable Bacillus subtillis, Staphylococcus aureus, Bacillus Cereus, Micrococcus luteus, Escherichia coli, Proteus vulgaris, Pseudomonas aeruginosa, Salmonella typhi organisms per $\mathrm{mL}$ of the stock suspensions was determined by means of the surface viable counting technique. About $\left(10^{8}-10^{9}\right)$ colonyforming units per $\mathrm{mL}$ was used. Each time, a fresh stock suspension was prepared; the experimental conditions were maintained constant so that suspensions with very close viable counts would be obtained.
Preparation of standard fungal suspensions: The fungal cultures Aspergillus niger, Candida albicans were maintained on Saboraud dextrose agar, incubated at $25^{\circ} \mathrm{C}$ for 4 days. The fungal growth was harvested and washed with sterile normal saline and finally suspended in $(100 \mathrm{~mL})$ of sterile normal saline and the suspension was stored in refrigerator till used.

In vitro testing of extracts for antimicrobial activity: Testing for antibacterial activity: The cup-plate agar diffusion method was adopted according to (Kavanagh, 1972) to assess the antibacterial activity of the prepared extracts. $0.6 \mathrm{~mL}$ of standardized bacterial stock suspensions (10 -10) colony- forming units per $\mathrm{mL}$ was thoroughly mixed with $60 \mathrm{~mL}$ of sterile nutrient agar. 20 $\mathrm{mL}$ of the inoculated nutrient agar were distributed into sterile Petri dishes. The agar was left to set and in each of these plates 4 cups, $10 \mathrm{~mm}$ in diameter, were cut using a sterile cork borer no. 4 and the agar discs were removed. Alternate cups were filled with $0.1 \mathrm{~mL}$ of each extracts using micro titer-pipette and allowed to diffuse at room temperature for $2 \mathrm{~h}$. The plates were then incubated in the upright position at $37^{\circ} \mathrm{C}$ for $18 \mathrm{~h}$. Two replicates were carried out for each extract against each of the test organism. Simultaneously addition of the respective solvents instead of extracts was carried out as controls. After incubation the diameters of the results and growth inhibition zones were measured, averaged and the mean values were tabulated.

Testing for anti-fungal activity: The same method as for bacteria was adopted. Instead of nutrient agar, yeast and Mould extract agar was used. The inoculated medium was incubated at $25^{\circ} \mathrm{C}$ for two days for the Candida albicans and three days for Aspergillus niger. (Lutterodt et al., 1999)

\section{Wound healing activity:}

Animals: Wistar albino rats of either sex weighing between 180 and $200 \mathrm{~g}$ were obtained from Nitin Biologicals, New Delhi, India the study was approved by the Institutional Ethics Committee for animal experimentation PDM School of Pharmacy, Safidon (PDM/IEAC/11/06/02), Safidon, Haryana (India) and all the procedures on animals were carried out as per CPCSEA guidelines, India. The animals were acclimatized to standard laboratory conditions of temperature $\left(22 \pm 3^{\circ} \mathrm{C}\right)$ and maintained on $12: 12 \mathrm{~h}$ light: dark cycle. They were provided with regular rat chow (VRK laboratory animal feed) and distilled water ad libitum. 
Am. J. Pharm. \& Toxicol., 6 (4): 119-123, 2011

Acute skin irritation test: The study was carried out as suggested by (Gfeller et al., 1985) on rats. An area measuring about $500 \mathrm{~mm}^{-2}$ on the dorsal fur of the animals was shaved. The extracts were applied separately to different groups of animals. After $4 \mathrm{~h}$, the skins were observed for signs of inflammation.

Excision wound model: Excision wounds were created by excising a circular piece $\left(500 \mathrm{~mm}^{2}\right.$ in areas) of full thickness skin from the dorsal interscapular region (Bairy and Rao, 2001). Wound contraction was monitored by measuring wound area, planimetrically, on alternate days till the wounds were completely healed. This was expressed as percentage of wound contraction. Time taken for complete epithelialization was noted by recording the days required for fall of scab leaving no raw wound behind.

Incision wound model: All animals were anaesthetized before wound creation and two paravertebral long incisions were made through the skin at the distance of about $1.5 \mathrm{~cm}$ from midline on each side of the depilated back of rat. The both edges kept together and stitched with black silk surgical thread (no. 000) and a curved needle (no. 11). The continuous threads on both wound edges were tightened for good closure of the wound. After stitching, extract and dexamethasone were applied daily and on alternate days respectively up to 9 days; when wounds were cured thoroughly the sutures were removed on the day 9 and tensile strength of cured wound skin was measured using tensiometer (Hemalata et al., 2001).

Statistical analysis: The data obtained by the various parameters was statistically evaluated by one way analysis of variance (ANOVA) followed by dunnet's t test using Graph Pad Prism software (Graph Pad software Inc., Version 4.0.0.255). The mean values \pm SEM were calculated for each parameter. The differences and the changes in healing by the plant extracts treated groups against the control group and standard group were analyzed accordingly. Level of significance was kept at $\mathrm{p}<0.05$.

\section{RESULTS}

Phytochemical screening of V. Vitigenia L. leaves Preliminary phytochemical screening of the ethanolic and aqueous extracts showed the presence of terpenoids, saponins, tannins and flavonoids.
Table 1: Antimicrobial activities of $10 \mathrm{mg} \mathrm{mL}^{-1}$ of $V$. Vitigenia $\mathrm{L}$. extracts (minimum inhibitory concentration)

\begin{tabular}{lllll}
\hline & & \multicolumn{2}{l}{ Zone of inhibition $(\mathrm{mm})$} \\
Strain & Organism & Standard & Alcoholic & Aqueous \\
\hline Gram positive & Bacillus subtillis & 23 & 15 & 17 \\
Gram positive & Staphylococcus aureus & 21 & 14 & 16 \\
Gram positive & Bacillus Cereus & 30 & 14 & 16 \\
Gram positive & Micrococcus luteus & 31 & 21 & 22 \\
Gram negative & Escherichia coli & 30 & 13 & 21 \\
Gram negative & Proteus vulgaris & 32 & 20 & 23 \\
Gram negative & Pseudomonas aeruginosa & 30 & 19 & 22 \\
Gram negative & Salmonella typhi & 23 & 15 & 16 \\
Fungal & Aspergillus niger & 30 & 18 & 23 \\
Fungal & Candida albicans & 20 & 17 & 18 \\
\hline
\end{tabular}

Table 2: Percentage wound contraction (Mean \pm SEM)

\begin{tabular}{lc}
\hline Group & $\begin{array}{l}\text { Percentage wound contraction } \\
\text { on 13th day (Mean } \pm \text { SEM) }\end{array}$ \\
\hline Control group & $64.7 \pm 1.20000$ \\
Standard group & $75.45^{* *} \pm 1.760$ \\
Aqueous extract & $95.03^{* * *} \pm 1.95$ \\
Ethanolic extract & $94.48 * * * \pm 1.35$ \\
\hline
\end{tabular}

Table 3: Period of epithelialization (Mean \pm SEM)

\begin{tabular}{lr}
\hline Groups & $\begin{array}{l}\text { Period of epithelialization } \\
\text { (Mean } \pm \text { SEM) (In days) }\end{array}$ \\
\hline Control group & $23.33 \pm 0.33000$ \\
Standard group & $19.66^{* *} \pm 0.330$ \\
Aqueous extract & $13.33^{* * *} \pm 0.33$ \\
Ethanolic extract & $14.66^{* * *} \pm 0.33$ \\
\hline
\end{tabular}

Antimicrobial activity: For bacterial strain Gentamycine $50 \mu \mathrm{g} \mathrm{mL} \mathrm{mL}^{-1}$ and for fungal strain Amphotericine B $50 \mu \mathrm{g} \mathrm{mL}^{-1}$ is used. Both the plant extracts show good antimicrobial activity against Micrococcus luteus, Escherichia coli, Pseudomonas aeruginosa, Proteus vulgaris, Aspergillus niger strains. Proteus vulgaris mainly cause the wound infection and these both the extracts showed good antimicrobial activity. It was observed that aqueous extract showed good activity as compared to that of ethanolic extract. Both the extracts showed better activity in gm-ve bacteria (Table 1).

\section{Wound healing study:}

Acute skin irritation test: In the skin irritation study, the tested extracts did not showed any type of irritation and there was no evidence of any noticeable inflammation on the skin.

Wound contraction: On day 4, the wound contraction of standard and extract treated groups was found to be significant $(\mathrm{p}<0.05)$ in comparison to control group. On day 13 , aqueous extract treated wound was completely healed while ethanolic extract treated group was also almost at complete healing stage. On day 18, standard ointment treated group healed $100 \%$ and control group showed $95.71 \%$ healing. It was also observed that epithelialization period of treated and standard groups were less in comparison to control group. Results are shown in Table 2 and 3 and Fig. 1-3. 


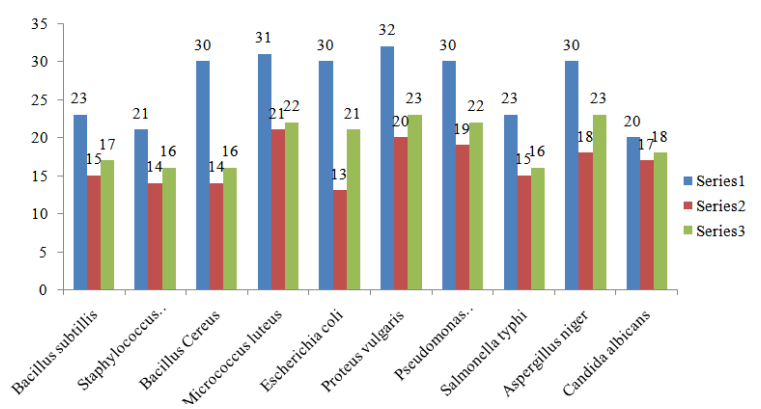

Fig. 1: Minimum inhibitory concentration

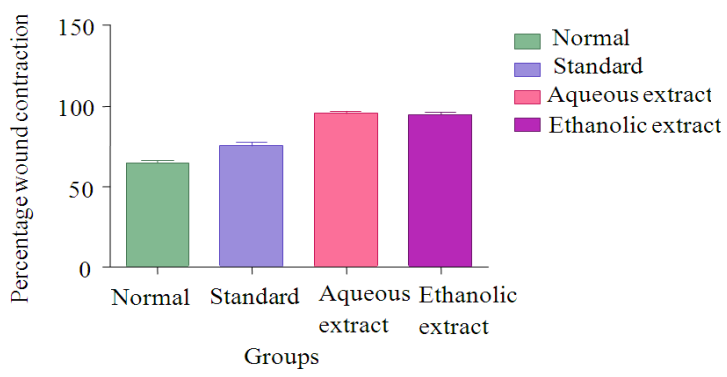

Fig. 2: Percentage wound contraction in excision wound model

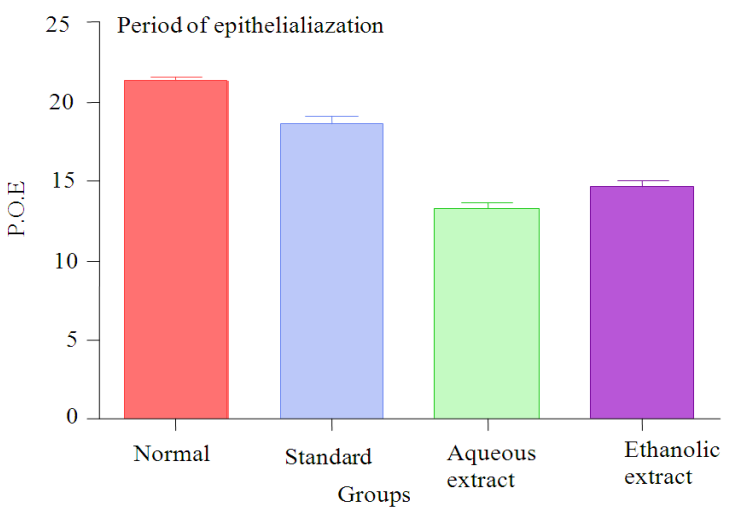

Fig. 3: Period of epithelialization in excision wound model

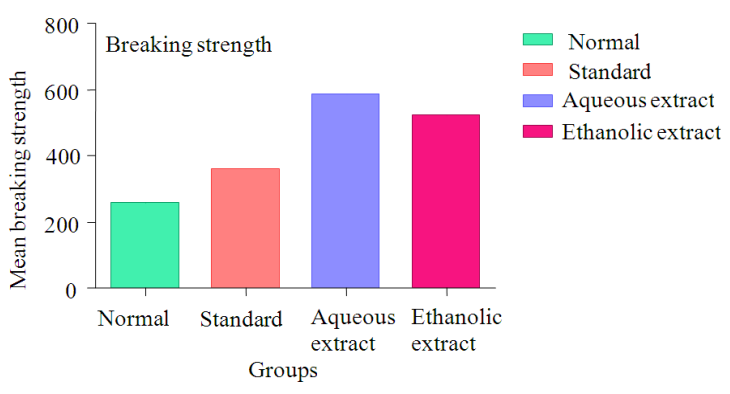

Fig. 4: Breaking strength in incision wound model
Table 4: Breaking strength (Mean \pm SEM)

\begin{tabular}{ll}
\hline Groups & $\begin{array}{l}\text { Breaking strength } \\
\left(\text { Mean } \pm \text { SEM) }\left(\mathrm{kg} \mathrm{cm}^{-2}\right)\right.\end{array}$ \\
\hline Control group & $258.33 \pm 1.66000$ \\
Standard group & $361.66 * * \pm 1.6600$ \\
Aqueous extract & $586.66 * * * \pm 1.66$ \\
Ethanolic extract & $521.66 * * * \pm 3.33$ \\
\hline
\end{tabular}

Tensile strength of incision wound model: The breaking strength of the incision wounds was increased in drug treated groups to significant extent, i.e., 586.66 $* * * \pm 1.66$ in aqueous extract and $521.66 * * * \pm 3.33$ in ethanolic extract treated group as compared to control group. The results are also comparable to standard drug Povidone iodine ointment.

Tensile strength for the treated group on 10th day was found to be very significant $(\mathrm{p}<0.001)$ than control group as shown in Table 4 and Fig. 4.

In incision wound model on 10th day, aqueous extract and ethanolic extract showed good result in comparison to standard and normal groups.

\section{DISCUSSION}

Phytochemical constituents such as tannins, flavonoids, alkaloids and several other aromatic compounds are secondary metabolites of plants that serve as defense mechanisms against predation by many microorganisms, insects and herbivores (Hemalata et al., 2001). The plant show the presence of tannins, saponins and flavonoids so this antimicrobial activity may be due to these phytoconstituents. This may therefore explain the demonstration of antimicrobial activity by the leaf extracts of $V$. Vitigenia $\mathrm{L}$. The demonstration of antibacterial activity against both gram positive and gram negative bacteria may be indicative of the presence of broad spectrum antibiotic compounds (Srinivasan et al., 2001).

Wound healing, a complex sequence of events, is initiated by the stimulus of injury to the tissues. A positive stimulus may result from the release of some factors by wounding of tissues. Cutaneous wound repair is accompanied by an ordered and definable sequence of biological events starting with wound closure and progressing to the repair and remodeling of damaged tissue. From the above result of excision wound model it is evident that on the day 13th day, there was significant increase in wound contraction in both the groups compared to control groups.

The tensile strength with incision model showed maximum activity for wound healing and the result was significant $(\mathrm{p}<0.01)$, i.e., $586.66 * * * \pm 1.66$ with aqueous extract and $521.66 * * * 3.33$ in ethanolic extract comparison to control. The V. Vitigenia leaf 
extract shows the presence of phytoconstituents like tannins, saponins and flavonoids are known to promote wound healing process. The study reveals that aqueous and ethanolic extract treated groups possesses good wound healing properties which may be attributed to the individual or combined action of phytoconstituents like tannins, saponins and flavanoids present in it. It can also be concluded that the maximum wound healing exerted by the aqueous extract of $V$. Vitigenia L. leaves.

\section{CONCLUSION}

This investigation has opened up the possibility of the use of this plant in drug development for human consumption possibly for the treatment of gastrointestinal, urinary tract and wound infections and typhoid fever .In conclusion, the results of study showed that the aqueous and alcoholic extract of V. Vitigenia L. effectively stimulates wound contraction in excision wound model and increase tensile strength of incision wounds as compared to control group and standard group. These finding could justify the inclusion of this plant in the management of wound healing.

\section{ACKNOWLEDGEMENT}

The researchers are grateful to Director Mr. Prit Pal Singh, PDM School of Pharmacy, Safidon, Jind (Haryana), India for the financial support and encouragement in carrying out this project.

Declaration of interest: The authors report no conflicts of interest. The authors alone are responsible for the content and writing of the study.

\section{REFERANCES}

Anonymous, B.P., 1994. General medical council, the pharmaceutical press. 17, Bloomsbury Square, London, W CI.P.
Bairy, K.L. and C.M. Rao, 2001. Wound healing profile of Ginkgo biloba. J. Natural Remedies, 1: 25-27.

Gfeller, W., W. Kobel and G. Seifert, 1985. Overview of animal test methods for skin irritation. Food Chem. Toxicol., 23: 165-168. DOI: 10.1016/02786915(85)90009-2

Hemalata, S., N. Subramanian, V. Ravichandran and K. Chinnaswamy, 2001. Wound healing activity of indigofera enneaphylla linn. Ind. J. Pharm. Sci., 63: 331-333.

Kavanagh, F., 1972. Analytical Microbiology. 1st Edn., Academic Press, New York, London, ISBN: 0124035027 , pp: 631.

Khandelwal, K.R., 2008. Practical Pharmacognosy. 1st Edn., Pragati Books Pvt. Ltd., Pune, ISBN: 8185790302, pp: 220.

Basu, B.D. and K.R. Kirtikar, 1999. Indian medicinal plants. 1st Edn., International Book Distributors, Dehra Dun.

Lutterodt, G.D., A. Ismail, R.H. Basheer and H.M. Baharudin, 1999. Antimicrobial effects of psidium guajava extract as one mechanism of its antidiarrhoeal action. Malaysian J. Med. Sci., 6: 17-20.

Cotran, R.S., V. Kumar, T. Collins and S.L. Robbins, 1999. Robbins Pathologic Basis of Disease. 6th Edn., Saunders, Philadelphia, London, ISBN: $072167335 \mathrm{X}, \mathrm{pp}: 1425$.

Srinivasan, D., S. Nathan, T. Sure and P.L. Perumalsamy, 2001. Antimicrobial activity of certain Indian medicinal plants used in folkloric medicine. J. Ethanopharmacol., 74: 217-220. DOI: 10.1016/S0378-8741(00)00345-7 